\title{
Adoption Intentions Toward Interactive Digital Television Among Advertising Professionals
}

\author{
Verolien Cauberghe
}

\author{
Ghent University
}

Patrick De Pelsmacker

University of Antwerp

\begin{abstract}
Using the decomposed theory of planned behavior, this article develops and empirically validates a model that predicts the intention to use interactive digital television as a marketing communication medium. The study tests the model with samples of Belgian advertising professionals at two different moments in time. The results show that the impact of perceived usefulness on the intention to use interactive digital television is low compared with the impact of perceived ease of use and subjective norms. Over time, the compliance effect of external subjective norms on usage intention decreases, in favor of the internalization effect through perceived ease of use. Behavioral control and attitude have more impact in the second stage than in the first.
\end{abstract}

\section{Introduction}

Digitalization has changed the media landscape by blurring the lines between existing forms (Edelman 2007). For example, interactive digital television (IDTV) is an outcome of this convergence that merges traditional broadcasting and digital (telecommunication) technology to allow viewers to interact with the television, using remote controls.

For this research, we define IDTV as (1) a group of technologies that gives (2) users the (3) possibility to take control (4) over their TV experience, (5) enabling interactivity with the content (6) (in)dependently of the distribution channel. First, IDTV encompasses a group of technologies, such as the television screen, a set-top box, personal video recorders (PVR), interactive program guides, and the remote control, rather than representing a single advance. Second, rather than viewers, people who consume IDTV are users, who can employ their televisions for various activities, such as banking, e-mailing, gaming, watching television, or playing along with a televised game. Third, the user is not required to take control over the television; it is possible to use IDTV in a traditional, linear way. However, he or she may choose what to watch and when to watch it by employing a PVR or video on demand (VOD) services. Fourth, the experience of using IDTV extends traditional television viewing. Fifth, this experience includes all three aspects of interactivity (McMillan and Hwang 2002), namely, user control, two-way communication, and synchronization. Sixth, IDTV is a new medium that may be broadcast through different platforms, including terrestrial (DVB-T), satellite (DVB-S), telephone cable (DVB-C), or broadband (IPTV), such that it could be 
independent or dependent of the channel. We also acknowledge that digital broadcast channels can be transmitted through satellite and terrestrial infrastructures, though these methods do not allow for real two-way communication, because there is no means for immediate feedback. In these scenarios, short messaging, e-mails, and telephone contacts must serve as feedback channels.

We focus on IDTV delivered through digital cable systems, which support immediate interactivity and feedback. In Belgium, compared with other European countries and the United States, IDTV adoption has been relatively slow, though cable penetration is nearly $100 \%$, so viewers already have access to broad channel choices. In 2009, IDTV penetration in Europe was $69.2 \%$, with Finland as the leading market (100\% penetration), followed by the United Kingdom with $90 \%$, Norway with $83 \%$, France with $81 \%$, and Spain with $80 \%$ penetration. In Belgium though, only $47 \%$ of consumers had adopted IDTV in 2009 (E-Media Institute 2009).

Interactive digital television also offers new advertising opportunities and formats (Cauberghe and De Pelsmacker 2006), with some pertinent threats to advertising professionals, such as the potential for ad skipping (e.g., Fortunato and Windels 2005) and unstable business models. Broadcasters thus worry that new entrants (e.g., content producers, software producers) might deliver content directly to the telecommunication providers and bypass their packaging function (Pagani 2000). In response to the business concerns initiated by new digital technologies, including VOD and PVR, Wirtz and Schwartz (2001) recommend that broadcasters cooperate and embrace these new developments (Cauberghe and De Pelsmacker 2006). Yet even as IDTV continues to spread worldwide, advertising professionals remain skeptical about investing in it (Tauder 2005).

We consider the interdependence of broadcasters, advertisers, and end users and suggest that the adoption of IDTV by advertisers is crucial (Leckenby 2003). In turn, we attempt with this study to gain insight into what drives advertising professionals to use or invest in IDTV advertising by building and empirically validating a predictive model of adoption intentions, using Belgian data. The unit of analysis is the person in an organization in charge of buying, budgeting, employing, or outsourcing the advertising budget. Using a decomposed theory of planned behavior framework (Taylor and Todd 1995), we also examine intentions to use IDTV among advertising professionals. Attitudes and intentions can change over time, so we collect two measurements at different moments in time, though both take place during a preadoption phase. Therefore, we can explore how intentions form among advertising professionals with regard to the use of interactive digital television over time.

\section{Conceptual Framework}

Many researchers use the theory of planned behavior (Ajzen 1991) to predict individual acceptance of information technology (e.g., Hsu, Lu, and Hsu 2007; Hung and Chang 2005; $\mathrm{Wu}$ and Chen 2005). According to this theory, behavioral intentions can predict actual behavior (Sheppard, Hartwick, and Warshaw 1988). The theory of planned behavior also states that behavioral intentions reflect three determinants: a person's attitude toward the behavior, subjective norms, and perceived behavioral control. Cognitive beliefs about the object of the behavior and their respective importance influence these three dimensions.

Although the theory of planned behavior has good explanatory power for predicting and understanding future behavior, it also suffers some practical and theoretical disadvantages 
(Taylor and Todd 1995). In particular, it combines cognitive belief structures into onedimensional constructs (attitudes, subjective norms, perceived behavioral control), which prevents any determination of the relative importance of each cognitive belief. Therefore, Taylor and Todd (1995) decompose the extended the theory of planned behavior into sets of salient beliefs.

The decomposed theory of planned behavior thus defines three antecedents of attitude: perceived ease of use (PEOU), perceived usefulness (PU), and compatibility (Moore and Benbasat 1991; Taylor and Todd 1995; Torznatzky and Fleischer 1990). Perceived ease of use is "the degree to which a person believes that using the system will be effortless" (Davis 1989, p. 320), and PU is "the degree to which a person believes that using a particular technology will enhance his/her job performance" (Plouffe, Hulland, and Vandenbosch 2001, p. 212). Compatibility refers to the degree to which the innovation fits with the potential adopter's existing values, previous experiences, and current needs (Rogers 1995).

Furthermore, different reference groups influence subjective norms, namely, peers, superiors, and subordinates. The direct effect of subjective norms on behavioral intentions is called the "compliance effect" (Venkatesh and Davis 2000), such that people choose to perform an action when one or more important people in their environment say they should. Venkatesh and Davis (2000) argue that subjective norms also have indirect effects on behavioral intentions through PU and PEOU. This "internalization process" thus represents the tendency of people to believe information provided by others.

Finally, the internal notion of individual self-efficacy (Bandura 1986) relates to perceived personal ability and external resource constraints and thus influences perceived behavioral control. The external resource constraints are similar to Triandis's (1977) facilitating conditions. In an organizational setting, they consist of resources (e.g., time, money) and technological compatibility.

We use an extended version of the decomposed theory of planned behavior to model the process by which usage intentions toward IDTV advertising form (see Figure1).

Figure 1. Conceptual Model of Adoption Intentions for Interactive Digital Television Advertising 


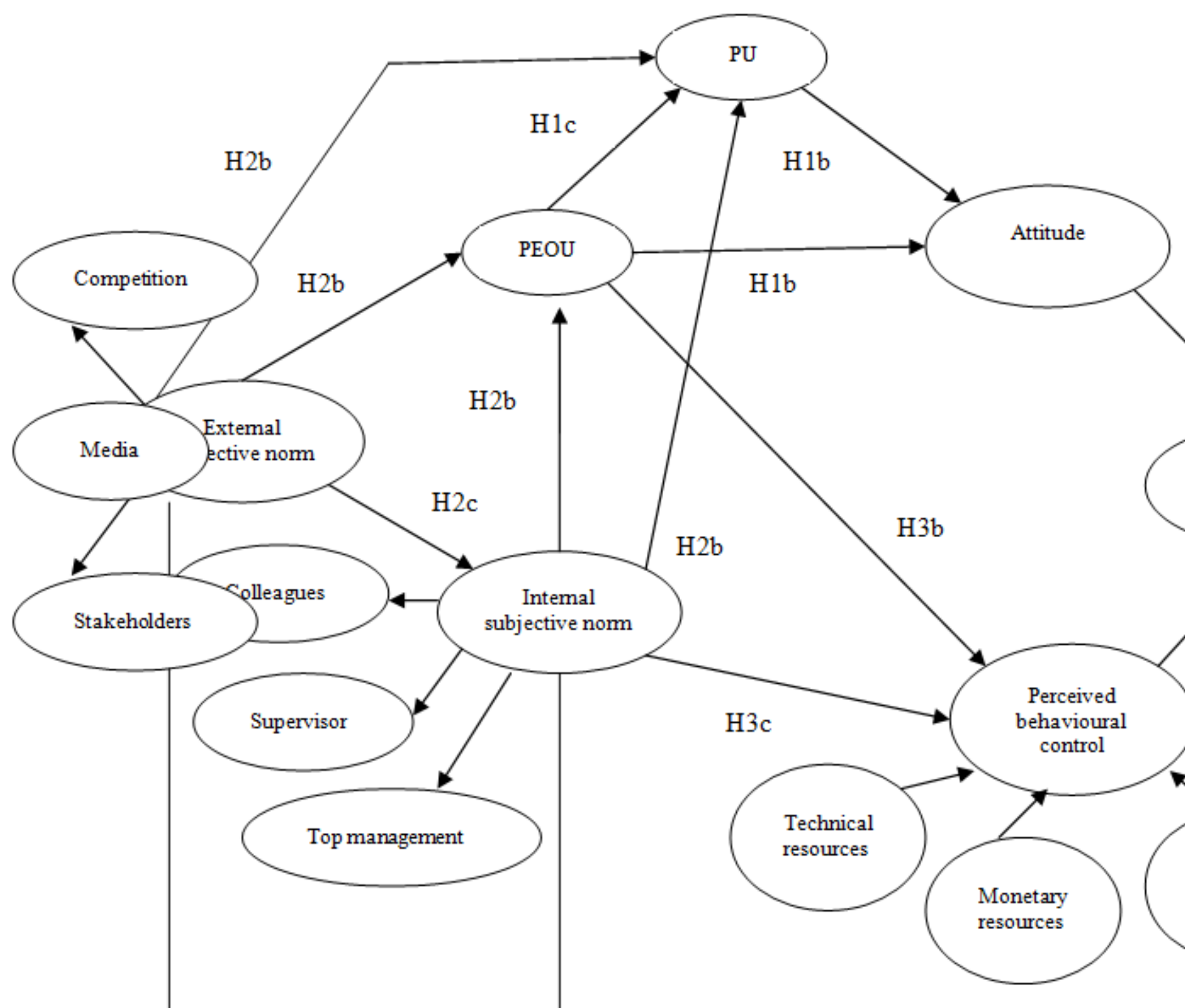

$\mathrm{H} 2 \mathrm{a}$

$\mathrm{H} 2 \mathrm{a}$

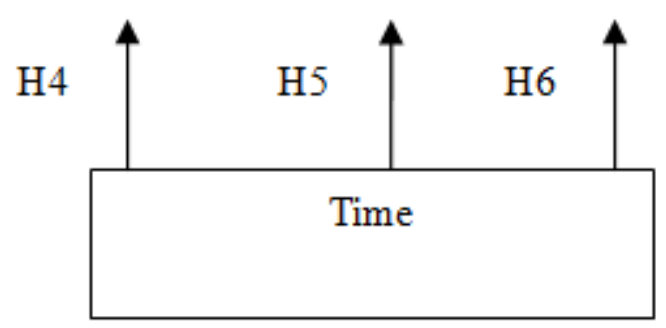

\section{Hypotheses}




\section{Attitudes}

Most user acceptance studies (for an overview, see Venkatesh et al. 2003) indicate a positive effect of attitude on behavioral intentions. In the decomposed theory of planned behavior, perceived usefulness and perceived ease of use are cognitive belief antecedents of this general attitude. However, compatibility does not appear in the model, considering its conceptual and empirical overlap with PU (e.g., Karahanna, Argarwal, and Angst 2006). Sun and Zhang (2006) undertake a meta-analysis and conclude that PU has a significant effect on attitude. Most studies also confirm a positive effect of PEOU on attitude, though in another metaanalysis, Schepers and Wetzels (2007) find that the effect of PU is much stronger than that of PEOU. Perceived ease of use also has a positive and significant effect on PU, according to most studies (e.g., Avlonitis and Panagopoulos 2005). Therefore, we predict:

H1a: Attitude toward IDTV advertising has a positive effect on the intention to adopt IDTV advertising.

H1b: Perceived usefulness and perceived ease of use of IDTV advertising have a positive effect on attitude toward using IDTV advertising.

H1c: Perceived ease of use of IDTV advertising has a positive effect on perceived usefulness of IDTV advertising.

\section{Subjective Norms}

In mandatory use contexts, in which people must use a tool or object (e.g., organizational settings), the effect of subjective norms on behavioral intentions is more substantial than in voluntary contexts (e.g., Workman 2005). However, different reference groups may have different impacts on the adoption process (e.g., Hung and Chang 2005). Following Tornaztzky and Fleischer's (1990) technology-organization-environment classification, we divide subjective normative influences on advertising professionals into two dimensions: an external, environmental, subjective norm and an internal, organizational, subjective norm. These dimension have potentially different influences in the model and thus are explored as separate factors.

Three reference groups determine external subjective norms: stakeholders (Riemenschneider, Harrison, and Mykytyn 2003), competitors (Frambach and Schillewaert 2002;

Papastathopoulou, Avlonitis, and Panagopoulos 2007), and media (Rogers 1995). The internal subjective norm instead comprises the following reference groups: top management (e.g., Tarafdar and Vaidya 2006; Taylor and Todd 1995), direct supervisors (e.g., Avlonitis and Panagopoulos 2005), and colleagues (Taylor and Todd 1995).

The compliance effect suggests a direct impact of both internal and external subjective norms on behavioral intentions to use IDTV advertising. The internalization effect also implies that the opinion of important reference groups will influence people's perceptions of the usefulness and ease of use of this tactic, because subjective norms constitute one of the most influential antecedents of PU and PEOU (Schepers and Wetzels 2007; Sun and Zhang 2006).

Rogers (1995) also claims that in the pre-adoption period, mass media and external communication have important roles in diffusing information about an offering and increasing awareness about it in the social network. For this study, we replace the external subjective 
norm of mass media with stakeholders and competition. In addition, the organization's top and middle management and employees learn about IDTV through not just mass media but also their interactions with professional associations, competitors, and technology stakeholders (e.g., Tarafdar and Vaidya 2006). Therefore, we anticipate that external reference groups have a significant effect on internal reference groups and hypothesize:

H2a: External and internal subjective norms have positive effects on the intention to adopt IDTV advertising.

H2b: External and internal subjective norms have positive effects on perceived usefulness and perceived ease of use of IDTV advertising.

H2c: External subjective norms have positive effects on internal subjective norms with respect to the adoption of IDTV advertising.

\section{Perceived Behavioral Control}

According to the decomposed theory of planned behavior, self-efficacy and facilitating factors influence perceived behavioral control (Taylor and Todd 1995). Most studies find a positive effect of perceived behavioral control (PBC) on behavioral intentions. In addition, Pavlou and Fygenson (2006) indicate that the perceived ease of use of searching for online information and making an online purchase correlates highly with the PBC of both behaviors $(\mathrm{r}=.43, \mathrm{r}=$ .52 , respectively). Other researchers similarly reveal strong correlations between PEOU and PBC (e.g., Yi et al. 2005).

In an organizational setting, top and middle management must provide the resources needed for employees to adopt and use innovations. For information technologies, management also plays a role in ensuring an adequate technological infrastructure. In their meta-analysis, Sabberwal, Jeyaraj, and Chowa (2006) find that management support is positively associated with facilitating conditions (e.g., resources, technological ability). Because personal ability is another dimension of PBC, we predict peers and colleagues also have an influence on this dimension.

H3a: Perceived behavioral control has a positive effect on the intention to adopt IDTV advertising.

H3b: Perceived ease of use of IDTV advertising has a positive effect on perceived behavioral control toward IDTV advertising.

H3c: Internal subjective norms have positive effects on perceived behavioral control toward IDTV advertising.

\section{Time as a Moderator}

Although many researchers have tried to examine the antecedents of adoption behavior empirically and conceptualize a multiphase, sequential adoption process, longitudinal studies that examine the potentially different influences of these antecedents remain relatively scarce (Karahanna, Straub, and Chervany 1999). The few longitudinal studies available indicate that the elements of the theory of planned behavior shift in their impacts on behavioral intentions, according to the different adoption stages (Thompson, Higgins, and Howell1994). Most 
studies investigate the effects of direct experience and consider the differences in cognitive beliefs that determine adoption decisions (pre-adoption) versus those that influence continuing usage (post-adoption) (e.g., Bhattacherjee and Premkumar 2004; Hsu, Lu, and Hsu 2007; Hu, Clark, and Ma 2003; Kim and Malhotra 2005; Lam and Lee 2002). However, in 2006-2007, when we conducted this study, IDTV was in the early diffusion stage in Belgium, so we examine how the determinants of intentions to use evolve over time within the preadoption stage. Unlike other longitudinal studies of technology acceptance, none of the advertising professionals in our study have direct experience with IDTV. Therefore, we conducted wave 1 in May 2006, six months after the commercial launch of IDTV by the two major telecommunication providers in Belgium (Telenet and Belgacom), and wave 2 followed a year later.

In general, longitudinal studies find support for a declining influence of subjective norms over time. Venkatesh and Davis (2000) theorize that people initially know little about an innovation, so subjective norms provide them with information that has an important direct compliance effect on behavioral intentions. Rogers (1995) similarly emphasizes the importance of communication in the early phase of the diffusion process. However, the two dimensions of subjective norms, internal and external, likely have different impacts on adoption intentions. Deutsch and Gerard (1955) also distinguish two types of social influence: informational social influence, or "the desire to be right," and normative social influence, or "the desire to be liked" (Fein, Goethals, and Kugler 2007, p. 167).

The external subjective norm exerts pressure on advertising professionals to use the innovation by informing them about it. This information can invoke their desire to be right. The external subjective norm also could influence their understanding of the image of the advertising professional and thus their desire to be liked, as evoked by the social prestige surrounding the innovation. Within an organization, the desire to be liked may be even greater than the desire to be right. In contrast, the internal subjective norm may be less important as an information source. Over time though, the advertising professional gathers sufficient information, and the informational social influence evoked by the external subjective norm should decrease. The influence of normative social influence instead may persist, because status gains from innovation use likely continue. Therefore, we predict that the influence of the external subjective norm on behavioral intentions decrease but does not disappear, whereas the influence of the internal subjective norm remains stable.

Over time, people also internalizes outside opinions, which implies a shift from the compliance effect to the internationalization effect. Therefore, the direct effect of subjective norms on intentions may diminish over time, but its effect through perceived usefulness and perceived ease of use should increase (e.g., Morris and Venkatesh 2000; Venkatesh et al. 2003). We do not make any predictions about different patterns for external or internal subjective norm influences on PU and PEOU over time.

H4a: The positive effect of the external subjective norm on the intention to adopt IDTV advertising is weaker in wave 2 than in wave 1 . The strength of the positive effect of the internal subjective norm on the intention to adopt IDTV advertising does not differ between waves.

H4b: The positive effect of the external subjective norm on perceived usefulness and perceived ease of use of IDTV advertising is stronger in wave 2 than in wave 1 . 
H4c: The positive effect of the internal subjective norm on perceived usefulness and perceived ease of use of IDTV advertising is stronger in wave 2 than in wave 1.

Because the external subjective norm should have an important influence on PU and PEOU in wave 1 , then increase in wave 2 , we anticipate that advertising professionals are more informed about IDTV in wave 2 than in wave 1. Therefore, the effects of PU and PEOU on attitude should increase over time.

H5: The positive effect of perceived usefulness and perceived ease of use of IDTV advertising on attitude toward adopting IDTV advertising is stronger in wave 2 than in wave 1 .

Venkatesh et al. (2003) further find that facilitating conditions (i.e., PBC) have significant influences on intentions only in later stages of the adoption process. Therefore, we hypothesize:

H6: The positive effect of perceived behavioral control on the intention to adopt IDTV advertising is stronger in wave 2 than in wave 1.

\section{Method}

\section{Data Collection}

We used an online survey to collect the data. The sampling frame for both waves consisted of a database of marketing professionals and the staff of advertising and communication agencies. This database, which combines three databases of different professional marketing organizations, allowed us to identify 2,680 advertising professionals to receive an e-mail invitation with a link to the questionnaire. For the first wave, conducted in May 2006, or six months after the commercial launch of IDTV in Belgium, 437 advertising professionals (response rate $=16.3 \%$ ) completed the questionnaire. In the second wave, one year later (May 2007), the same sample frame received the same questionnaire, and the response rate was $16.7 \%(\mathrm{n}=448)$. The responses to both questionnaires were anonymous. The samples did not differ significantly with respect to the respondents' company type, function, responsibility, gender, age, or relevant work experience (see Table 1).To test for nonresponse bias, we compared the characteristics of the total sample frame with those of the sample and found no significant differences between groups.

Table 1. Comparison of Sample Characteristics: Waves 1 and 2

\begin{tabular}{|l|l|l|l|l|}
\hline & Wave 1 & Wave 2 & Chi $^{2}$ & $p$-Value \\
\hline Type of company & $11.2 \%$ & $11.4 \%$ & & \\
Advertising agency & $5 \%$ & $8.5 \%$ & & \\
Media planning & $83.8 \%$ & 80.1 & & \\
Advertiser & $10.5 \%$ & $9.6 \%$ & & \\
& & & & \\
\hline
\end{tabular}




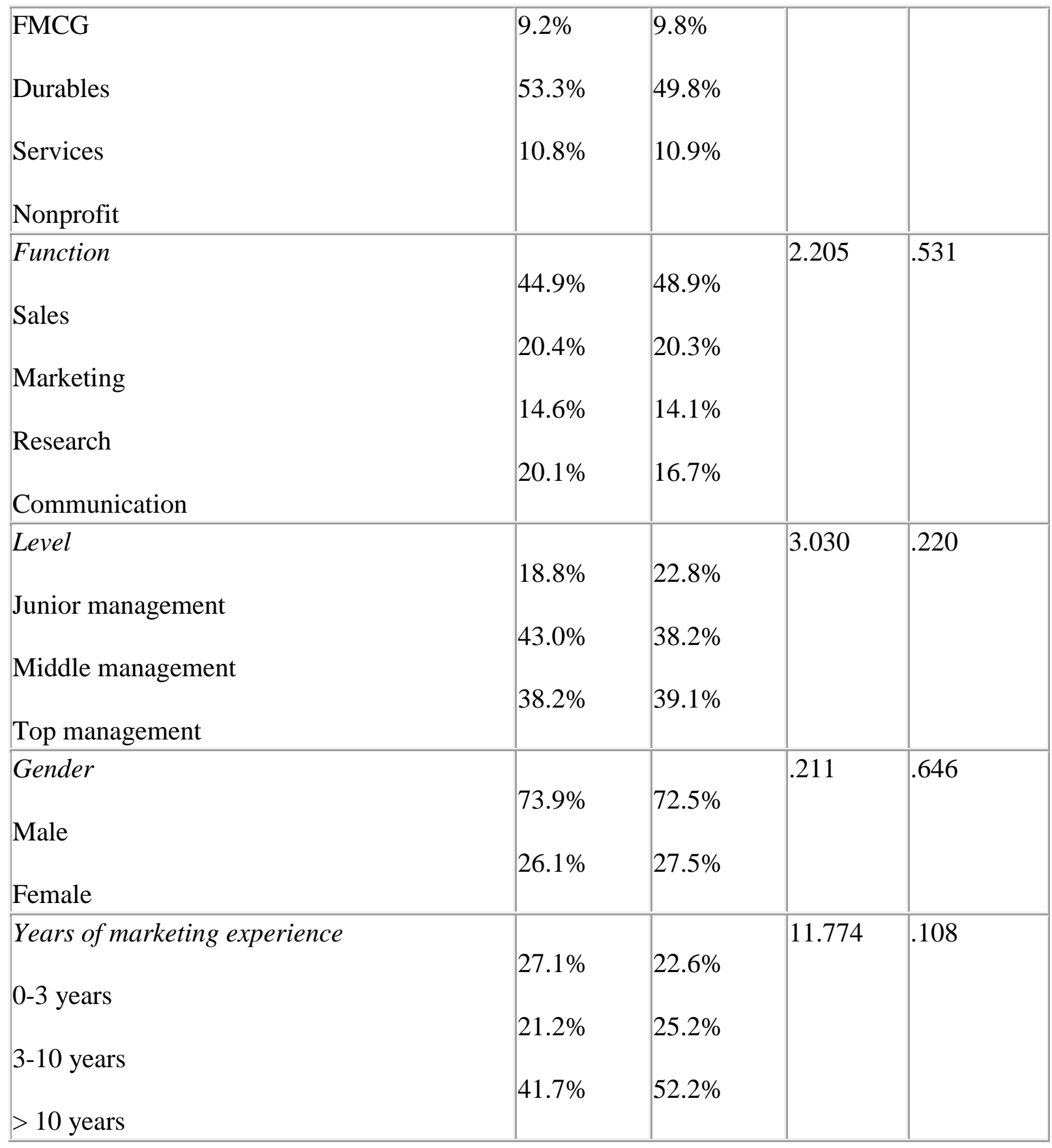

\section{Measures}

The questionnaire consists of measurement scales retrieved from marketing, management, and information systems literature. All constructs used five-point Likert scales (anchored at "strongly disagree" and "strongly agree") or semantic differential scales. The PU (e.g., "IDTV enhances my effectiveness as an advertising professional") and PEOU (e.g., "I would find IDTV easy to use in my communication strategy") measures came from Davis's (1989) scale. The attitude construct (e.g., "Using IDTV is a good/bad idea"); PBC (e.g., "I would be able to use IDTV as a marketing communication tool") as well as its antecedents, personal ability (e.g., "I would feel comfortable using IDTV on my own") and technological facilitating (e.g., "I will have trouble looking at my campaign on IDTV to test it"); and behavioral intentions (e.g., "I intend to use IDTV in the near future") all reflect the scales provided by Taylor and Todd (1995). 
External social influence includes three constructs. To measure the influence of media, we use Taylor and Todd's (1995) scale (e.g., "I read/saw new reports that using IDTV is a good medium to advertise"). The external subjective influence of competition features a scale developed by Avlonitis and Panagopoulos (2005) (e.g., "Competitive marketers are using IDTV during their contact with consumers"). For measuring the influence of stakeholders, we apply a newly developed scale that refers to the most important stakeholders for the IDTV business model in Belgium (e.g., Telecom company). Internal social influence also comprises three constructs. The influences of both top management (e.g., "The firm strongly encourages the use of IDTV for advertising purposes") and colleagues (e.g., "My colleagues are strongly encourages the use of IDTV for advertising purposes") use the scale provided by LeonardBarton and DeChamps (1988). The items to assess the normative influence imposed by the direct supervisor come from a scale by Avlonitis and Panagopoulos (2005) (e.g., "My supervisor continuously encourages me to use IDTV").

We conducted a pilot study to validate and clarify the measurement instruments. Of the 72 Belgian advertising professionals who received the questionnaire, 44 completed it (response rate $=61.1 \%$ ). On the basis of their responses, we removed or reformulated a few items.

\section{Results}

\section{Exploring the Factor Structure}

Levine and colleagues (2006) suggest that for established scales, confirmatory factor analyses are most appropriate. However, because not all the scales we used have been properly tested in prior studies, we first adopt exploratory factor analysis (principal components) with Varimax rotation to explore the factor structure of the constructs. All items except two load on their respective constructs. The three theorized internal subjective norm antecedents all load on one factor, and the items related to the two antecedents of perceived behavioral control load on another single factor. Therefore, we include the internal subjective norm and $\mathrm{PBC}$ in the model as general dimensions without antecedents.

\section{General Model}

The structural equation modeling module in SPSS, AMOS 6.0, can test general models (measurement and structural) using maximum likelihood estimation. The loadings, standardized residual covariances, and modification indices (Steenkamp and van Trijp 1991) identify any items that should be screened and removed.

The data for our study are not normally distributed (multivariate kurtosis $=339.905$; kurtosis = 90.901), so we use bootstrapping method (Bollen and Stine 1992) to estimate the fit. The general model achieves the cutoff values for all the fit indices (Bollen and Stine 1992; Hu and Bentler 1999) $\left(\mathrm{c}^{2}=953.568\right.$, degrees of freedom $[\mathrm{df}]=307, \mathrm{c}^{2} / \mathrm{df}=3.106$, Tucker-Lewis index $=.953$, confirmatory fit index $=.959$, root mean square error of approximation $=.049$ ). In addition to this satisfactory model fit, the measurement model possesses convergent validity, reliability, and discriminant validity (Bagozzi and Yi 1988). In the general model, the item loadings are all higher than the recommended cutoff value of .60 (Bagozzi and Yi 1988), in support of convergent validity. In addition, the average variance extracted of all latent constructs is greater than the threshold of .50, ranging between .54 and .82 (Bagozzi and Yi 1988). All Cronbach's alphas exceed .70, which implies a satisfactory level of reliability (Bagozzi and Yi 1988). The composite reliabilities, with values between .700 and .933, 
indicate the reliability and unidimensionality of the scales (Steenkamp and van Trijp 1991). We provide an overview of the reliability constructs in Table 2. Finally, we obtain evidence of discriminant validity from the correlations between the constructs, which all are significantly different from 1 (Singh and Rhoads 1991).

Table 2. Construct Reliability

\begin{tabular}{|l|l|l|l|}
\hline & $\begin{array}{l}\text { Cronbach's } \\
\text { Alpha }\end{array}$ & $\begin{array}{l}\text { Composite } \\
\text { Reliability }\end{array}$ & $\begin{array}{l}\text { Average Variance } \\
\text { Extracted }\end{array}$ \\
\hline Perceived usefulness & .878 & .878 & .71 \\
\hline Perceived ease of use & .716 & .70 & .54 \\
\hline Attitude & .801 & .801 & .57 \\
\hline External subjective norm & .896 & .845 & .65 \\
\hline Competition & .824 & & \\
\hline Stakeholders & .771 & & .78 \\
\hline Media & .908 & .912 & .62 \\
\hline Internal subjective norm & .871 & .871 & .82 \\
\hline $\begin{array}{l}\text { Perceived behavioral } \\
\text { control }\end{array}$ & .933 & .933 & \\
\hline Usage intention & & & \\
\hline
\end{tabular}

In Figure 2, we provide the structural paths of the final model and their significance. As expected, attitude has a positive effect on the intention to use IDTV advertising $(B=.264, p<$ .001 ), in support of H1a. However, perceived usefulness does not have a significant positive effect on attitude $(B=.09, p=.12)$, though we find that perceived ease of use has a strong positive effect on attitude $(\beta=.52, p<.001)$. Thus we find partial support for H1b. The effect of PEOU on PU also is positive, as we predicted in $\mathrm{H} 1 \mathrm{c}(\beta=.51, p<.001)$.

Figure 2. General Model with Structural Paths 


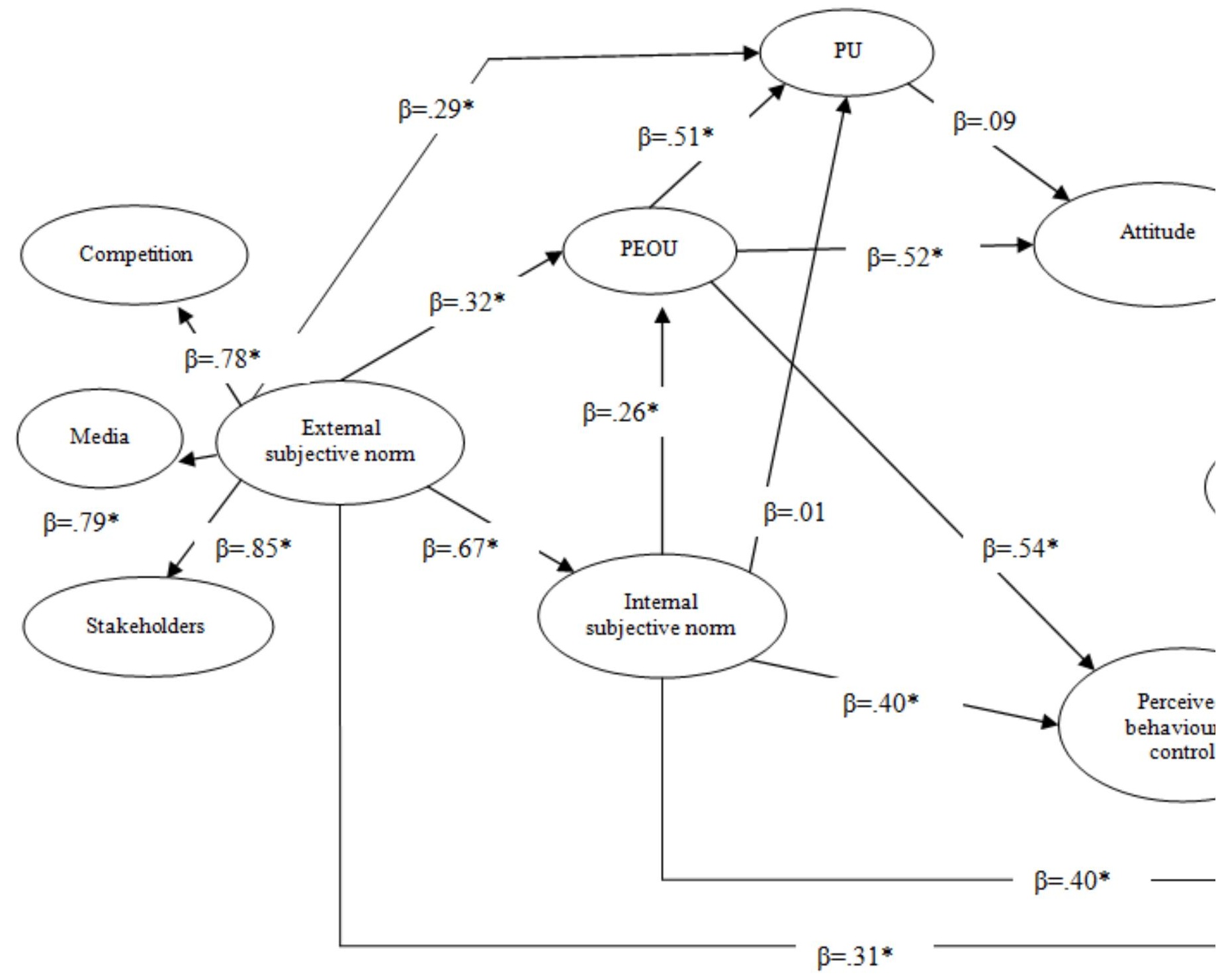

External subjective norms have significant positive effects on usage intention ( $\beta=.31, p<$ $.001)$, as do internal subjective norms ( $B=.40, p<.001)$, in support of H2a. These findings also reflect the compliance mechanism of subjective norms on adoption intention. The internalization effect of external subjective norms is also apparent. External subjective norms have significant effects on both PU $(\beta=.29, p<.001)$ and PEOU $(\beta=.32, p<.001)$. Whereas the effect of internal subjective norms on PU is not significant $(B=.01, p=.796)$, its effect on PEOU is significantly positive $(B=.26, p<.001)$. The results thus partly support $\mathrm{H} 2 \mathrm{~b}$. External subjective norms have a strong positive effect on internal subjective norms $(B=$ $.67, p<.001)$, in support of $\mathrm{H} 2 \mathrm{c}$.

The perception of being able to use IDTV advertising, or perceived behavioral control, has a positive effect on the intention to use it $(\beta=.22, p<.001)$, which supports H3a. Perceived ease of use also has a positive influence on $\mathrm{PBC}(\beta=.54, p<.001)$, which supports $\mathrm{H} 3 \mathrm{~b}$. Consistent with $\mathrm{H} 3 \mathrm{c}$, the effect of internal subjective norms on $\mathrm{PBC}$ is positive $(B=.40, p<$ $.001)$. 
Finally, the reflective dimensions of the second-order construct, external subjective norms, all load highly on this latent construct (competition: $\beta=.78, p<.001$; media: $\beta=.79, p<.001$; stakeholders: $\beta=.85, p<.001)$.

\section{Comparison of Waves 1 and 2}

With a multiple group comparison analysis, we test if the structural paths of the hypothesized models differ between waves. By specifying nested models, we can identify any significant non-invariance between subsamples appears. In each of the nested models, we constrain a set of parameters to be equal in both subgroups. We then test this assumption by comparing the fit of the nested models with the fit of the general model. If any decline in fit is substantial, the constraints must be rejected, and the parameters are different (non-invariant) for the subgroups. The first step in multiple group comparison analysis thus is the test for metric invariance, a prerequisite of structural invariance (Byrne 2001), which requires keeping all factors or measurement loadings equal across groups. The results indicate the invariance of the measurement model for the subgroups of waves 1 and $2\left(\Delta \chi^{2}=13.969, \Delta \mathrm{df}=18, p=\right.$ .731). The second step investigates structural invariance, and we find significant variance for the structural weights across the two waves $\left(\Delta \chi^{2}=34.728, \Delta \mathrm{df}=16, p=.004\right)$.

To identify which structural path weights are not equal across time, we performs an iterative analysis in which we constrain, then set free, each separate relationship. The results indicate that several paths are significantly different across groups.

We anticipated that the influence of external subjective norms on advertising professionals' intentions to use IDTV as a marketing communication tool would diminish in wave 2 compared with wave 1 . The results confirm this expectation ( $\beta$ wave $1=.41, p<.001 ; \beta$ wave $2=.22, p=.001 ; \Delta \chi^{2}=4.940, \Delta p=.026$ ). For internal subjective norms, the influence on behavioral intentions remains stable over time, as expected ( $\beta$ wave $1=.25, p<.001$; $\beta$ wave $\left.2=.24, p<.001 ; \Delta \chi^{2}=.031, \Delta p=.860\right)$, in support of H4a. The effect of external subjective norms on PU is positive but does not increase over time ( $\beta$ wave $1=.35, p<.001$; $\beta$ wave $2=$ $.23, p=.002 ; \Delta \chi^{2}=1.034, \Delta p=.309$ ), whereas its effect on PEOU is stronger in wave 2 than in wave 1 ( $\beta$ wave $1=.20, p=.021 ; \beta$ wave $2=.42, p<.001 ; \Delta \chi^{2}=4.239, \Delta p=.040$ ). We thus find support for $\mathrm{H} 4 \mathrm{~b}$. Internal subjective norms have no significant effects on PU in wave 1 or wave 2 ( $\beta$ wave $1=.002, p=.979 ; \beta$ wave $2=.009, p=.883 ; \Delta \chi^{2}=.003, \Delta p=.954$ ). Instead of an increase in the positive influence of internal subjective norms on PEOU, we find a stable positive effect over time ( $\beta$ wave $1=.31, p<.001 ; \beta$ wave $2=.21, p=.005 ; \Delta \chi^{2}=$ $.445, \Delta p=.505$ ), which conflicts with $\mathrm{H} 4 \mathrm{c}$. The positive effects of both PU and PEOU on attitude reveals that though the effect of the former is positive in wave 1 , it becomes significantly weaker and even insignificant in wave 2 ( $\beta$ wave $1=.23, p<.001 ; \beta$ wave $2=-$ $\left..08, p=.304 ; \Delta \chi^{2}=7.282, \Delta p=.007\right)$. For the latter, the results are in line with our expectations: Perceived ease of use has a stronger effect on attitude in wave 2 than in wave 1 ( $\beta$ wave $1=.36, p<.001 ; \beta$ wave $2=.68, p<.001 ; \Delta \chi^{2}=5.333, \Delta p=.021$ ). The results thus partially support $\mathrm{H} 5$.

Perceived behavioral control has a small influence on behavioral intentions in wave 1 , and its effect increases significantly in wave 2 ( $\beta$ wave $1=.15, p=.008$; $\beta$ wave $2=.29, p<.001$; $\left.\Delta \chi^{2}=4.632, \Delta p=.031\right)$, in support of H6.

The loadings of the second-order model of external subjective norms did not change significantly over time, which implies the stability of the second-order construct. 


\section{Discussion and Conclusions}

The adoption of interactive digital television by advertisers is important for the success of this medium. The main goal of this study therefore has been to detect, for the initial adoption stage, how the intention to use IDTV technology forms and how the relative importance of the determinants of such intention changes over time.

The model we tested explains approximately $63.5 \%$ of the variance of the behavioral attention to use IDTV as an advertising tool in wave 1 and $69.2 \%$ in wave 2, which is satisfactory. In the general model, the direct effect of external and internal subjective norms on this intention is stronger than the impact of the attitude toward using IDTV advertising or perceived behavioral control. Contrary to our expectations and the findings of past studies, the influence of perceived usefulness on the attitude toward adopting IDTV is insignificant. Prior studies have indicated that when people consider using a new technology, its perceived usefulness has a stronger influence than does its complexity (i.e., perceived ease of use) (Schepers and Wetzels 2007). Perhaps our results reflect the weak and somewhat negative impact of the first IDTV advertising campaigns launched by Belgian pioneers. In addition, technological hurdles in the Belgian IDTV infrastructure may have prompted advertising professionals to emphasize the importance of ease of use. In the general model, perceived ease of use thus plays a central role, strongly influencing perceived usefulness, attitude, and perceived behavioral control.

Beyond the strong compliance mechanism, both external and internal subjective norms also influence cognitive beliefs about IDTV as an advertising tool. For the former, this internalization effect is apparent for both PU and PEOU. The internal subjective norm only influences PEOU in relation to IDTV, not perceptions about its usefulness. Perhaps the internal reference groups themselves are not widely convinced of the usefulness of IDTV as an advertising tool.

The comparison of our results across waves indicates that the factors that influence the evaluation process differ over time. Although the direct impact of external subjective norms on behavioral intentions decreases over time, their effect on internal subjective norms and cognitive beliefs persists. The impacts of the attitude toward adopting IDTV advertising and of PBC on the intention to adopt both increase over time.

The effect of PU on the attitude toward adopting IDTV advertising is smaller in both wave 1 and wave 2 than the effect of PEOU on attitude. However, the effect of PEOU on PU remains stable over time, as expected. In wave 1, the internalization effect of external subjective norms is greater for PU than for PEOU. In wave 2, this balance shifts: The effect of external subjective norms on PEOU becomes important, but its effect on PU disappears. Previous longitudinal studies also indicate that the effect of PEOU diminishes over time, such that more versus less experienced users have a better opinion of the PEOU of an innovation (Venkatesh et al. 2003), which results in a lower direct impact on behavioral intentions and an indirect impact on PU (Szajna 1996). However, we examine changes in attitudes and beliefs only during the pre-adoption stage. Therefore, more information may have created a better impression of the PEOU of IDTV in the second wave. Only after the adoption and use of IDTV as an advertising tool should we expect the effect of PEOU to diminish.

In summary, the impact of PU on the intention formation process is low compared with the impact of PEOU and subjective norms. Over time, the compliance effect of external subjective norms (media, stakeholders, competition) on behavioral intentions decreases, in 
favor of the internalization effect through PEOU. Behavioral control and attitude have more impact in later than in earlier stages. The influence of internal subjective norms on behavioral intentions, through PBC, remains stable over time.

\section{Theoretical Contributions}

This study makes several contributions to innovation acceptance literature. First, we apply a well-established technology acceptance model, the decomposed theory of planned behavior, to the intention formation of advertising professionals with regard to using IDTV for their advertising. Second, we extend the decomposed theory of planned behavior by investigating the links across the three main model dimensions (attitude, subjective norms, perceived behavioral control) and their antecedents (cognitive beliefs). Prior research (Bagozzi 2007; Bagozzi and Lee 2002; Venkatesh and Davis 2000) stresses the mutual dependence of the main antecedents of intention, but Karahanna, Argarwal, and Angst (2006) claim past research has failed to confirm this dependence rigorously. Third, we identify two types of subjective norms with different influences on the usage intention of organizational members: external (environmental) influences and internal (organizational) influences (Tornatzky and Fleischer 1990). Fourth, this study measures usage intentions at two moments in time (2006 and 2007), after the introduction of IDTV.

In summary, our study provides insights into the drivers of the adoption process for IDTV advertising, using data from a country that introduced the technology to end customers. Motivating communication professionals to invest in this new marketing communication medium may indirectly lower the costs for end customers and thus lower barriers to popular adoption. However, even in countries with high customer adoption of IDTV, we find that communication professionals remain reluctant to invest in IDTV advertising. Because of their emphasis on return on investments, most communication professionals tend to rely on media they know, such as traditional television, press, and radio spots, instead of new media. The results of this study should encourage them to speed up their adoption process.

This study also is relevant, independent of its timing and technology. The insights provide added value for understanding the behavior of advertising professionals who face the adoption of a new technology or media format. Thus, for the early stages of an adoption process, it provides guidelines for implementing marketing communication innovations.

\section{Managerial Implications}

The study also offers insights to IDTV stakeholders regarding how to approach advertising professionals and increase their adoption behavior. Our results show that external subjective norms and perceived ease of use are the most important drivers. Positive media attention and the influence of stakeholders, such as through seminars, conferences, and presentations, are likely the best tools to convince advertisers of the potential of IDTV advertising. The strong effect of external subjective norms on internal subjective norms implies that top and middle managers should be the main target groups. Because we find no internalization effect of internal reference groups on perceived usefulness, we suggest managers should be persuaded about the positive benefits and usefulness of interactive digital television advertising as well.

Perceived ease of use has an important influence, so (mass) communication should focus on the user friendliness of advertising on IDTV. The declining effect of perceived usefulness on attitudes may be troubling for IDTV advocates though. Interactive digital television needs 
success stories from pioneers who are willing to try interactive campaigns. Such testimonies could increase perceptions of the usefulness of IDTV as an advertising tool. Therefore, offering free trials or cheap development costs could increase the willingness of advertisers to try IDTV formats.

Increasing adoption rates among end users also could force advertisers to include the medium in their marketing mix. Allen (1988) has argued that promotion strategies, such as subsidized pricing and free trials, were critical for the success of previous innovations. In many European countries, deliveries of free set-top boxes or discounts for digital decoders aim to boost the adoption of IDTV by end users.

\section{Limitations and Further Research}

This study suffers some limitations that suggest areas for further research. We did not incorporate the role of affect in the decision-making process of professionals within an organization (see Kim, Chan, and Chan 2007). A recurring problem in technology acceptance research, common method bias, also may be present in our study (Woszczynski and Whitman 2004). Because we use cross-sectional data in each wave, we cannot ascertain the direction of causality unambiguously. Although theory guided our hypotheses development, further research should confirm the causal directions by measuring different constructs at various moments in time. We examined advertising professionals' perceptions and behavioral intentions at two moments in time, but the data were not linked on an individual level, so we could not confirm individual changes over time. Further research might use a within-subjects design and incorporate feedback mechanisms that might influence reevaluations of an innovation (Kim and Malhotra 2005). In general, additional research on IDTV advertising usage should investigate the longitudinal character of the process by extending beyond the pre-adoption stage to the adoption decision and post-adoption behavior. Finally, potential moderating variables that might influence how the adoption process proceeds should be examined in further research, at both individual (e.g., personality traits such as innovativeness; Agarwal and Prasad 1998) and organizational (structure, culture, centralization, formalization) levels (Pelham and Wilson 1996).

\section{References}

Agarwal, Ritu, and Jayesh Prasad (1998), "A Conceptual and Operational Definition of Personal Innovativeness in the Domain of Information Technology," Information Systems Research, 9 (2), 204-215.

Allen, David (1988),"New Communications Services: Network Externalities and Critical Mass," Telecommunications Policy, 12 (3), 257-71.

Ajzen, Izen (1991), "The Theory of Planned Behavior," Organizational Behavior and Human Decision Processes, 50 (2), 179-211.

Avlonitis, George J. and Nikolaos G. Panagopoulos (2005), "Antecedents and Consequences of CRM Technology Acceptance in Sales Forces," Industrial Marketing Management, 34 (4), 355-68. 
Bagozzi, Richard P. (2007), "The Legacy of the Technology Acceptance Model and a Proposal for a Paradigm Shift," Journal of the Association of Information Systems, 8 (4), 24454.

--- and Kyu Hyun Lee (2002), "Multiple Routes for Social Influence: The Role of

Compliance, Internalization and Identity," Social Psychology Quarterly, 65 (3), 226-47.

--- and Youjae Yi (1988), "On the Evaluation of Structural Equation Models," Journal of the Academy of Marketing Science, 16, 74-94.

Bandura, Albert (1986), Social Foundations of Thought and Action. Englewood Cliffs, NJ: Prentice Hall.

Bhattacherjee, Anol, and G. Premkumar (2004). "Understanding Changes in Belief and Attitude toward Information Technology Usage: A Theoretical Model and Longitudinal Test," MIS Quarterly, 28 (2), 229-54.

Bollen, K.A. and R.A. Stine (1992), "Bootstrapping Goodness-of-Fit Measures in Structural Equation Models," Sociological Methods and Research, 21 (2), 205-29.

Byrne, B.M. (2001), Structural Equation Modeling with AMOS: Basic Concepts, Applications and Programming. Mahwah, NJ: Lawrence Erlbaum.

Cauberghe, Veroline and Patrick De Pelsmacker (2006), "Opportunities and Thresholds for Advertising on Interactive, Digital TV: A View from Advertising Professionals," Journal of Interactive Advertising, 7 (Fall), http://www.jiad.org/vol7/no1/cauberghe/index.htm (accessed November 13, 2009).

Davis, Fred D. (1989), "Perceived Usefulness, Perceived Ease of Use, and User Acceptance of Information Technology," MIS Quarterly, 13 (3), 319-39.

Deutsch, Morton and Harold B. Gerard (1955), "A Study of Informational and Normative Social Influence upon Individual Judgment," Journal of Abnormal and Social Psychology, 51 (3), 629-36.

Edelman, D.C. (2007), "From the Periphery to the Core: As Online Strategy Becomes Overall Strategy, Marketing Organizations and Agencies Will Never Be the Same," Journal of Advertising Research, 47 (2), 130-34.

E-Media Institute Europe (2009), "European Digital TV Market Map (W-Europe), Q3," EMedia Research report, December.

Fein, Steven, George R. Goethals, and Mattew B. Kugler (2007), "Social Influence on Political Judgments: The Case of Presidential Debates," Political Psychology, 28, 165-92.

Fortunato, John and Daniel M. Windels (2005), "Adoption of Digital Video Recorders and Advertising: Threats or Opportunities?" Journal of Interactive Advertising, 6 (1), http://www.jiad.org/issues (accessed September 2010). 
Frambach, Ruud T. and Niels Schillewaert (2002), "Organizational Innovation Adoption. A Multi-Level Framework of Determinants and Opportunities for Future Research," Journal of Business Research, 55 (2), 163-76.

Hsu, C.L., H.P. Lu, and H.H. Hsu (2007), "Adoption of the Mobile Internet: An Empirical Study of Multimedia Message Service (MMS)," Omega, 35 (6), 715-26.

$\mathrm{Hu}, \mathrm{Li}-\mathrm{Tze}$ and Peter M. Bentler (1999), "Cutoff Criteria for Fit Indexes in Covariance Structure Analysis: Conventional Criteria Versus New Alternatives," Structural Equation Modeling, 6 (1), 1-55.

Hu, Paul J., T.H.K. Clark, and W.W. Ma (2003), "Examining Technology Acceptance by School Teachers: A Longitudinal Study," Information and Management, 41 (2), 227-41.

Hung, Shin-Yuan and Chia-Ming Chang (2005), "User Acceptance of WAP Services: Test of Competing Theories," Computer Standards and Interfaces, 27 (4), 359-70.

Karahanna, Elena, Ritu Agarwal, and Corey M. Angst (2006), "Reconceptualizing Compatibility Beliefs in Technology Acceptance Research," MIS Quarterly, 30 (4), 781-804.

---, Detmar W. Straub, and Norman L. Chervany (1999), "Information Technology Adoption Across Time: A Cross-Sectional Comparison of Pre-Adoption and Post-Adoption Beliefs," MIS Quarterly, 23 (2), 183-213.

Kim, H-W., Hock Chuan Chan, and Y.P. Chan (2007), "A Balanced Thinking-Feelings Model of Information Systems Continuance," International Journal of Human-Computer Studies, 65 (6), 511-25.

Kim, Sung S. and Naresh K. Malhotra (2005), "A Longitudinal Model of Continued IS Use: An Integrative View of Four Mechanisms Underlying Postadoption Phenomena," Management Science, 51 (5), 741-55.

Lam, J.C.Y. and M.K.O. Lee (2002), "Digital Inclusiveness: Longitudinal Study of Internet Adoption by Older Adults," Journal of Management Information Systems, 22 (4), 177-206.

Leckenby, John D.(2003), "The Interaction of Traditional and New Media 2003," working paper, University of Texas at Austin.

Leonard-Barton, Dorothy and Isabelle Deschamps (1988), "Managerial Influence in the Implementation of New Technology," Management Science, 34 (10), 1252-65.

Levine, T.R., C. R. Hullet, Mitchell Turner, and Knight Lapinski (2006), "The Desirability of Using Confirmatory Factor Analysis on Published Scales," Communication Research Reports, 23, 309-14.

McMillan, Sally J. and Jang Sun Hwang (2002), "Measures of Perceived Interactivity: An Exploration of the Role of Direction of Communication, User Control, and Time in Shaping Perceptions of Interactivity,"Journal of Advertising, 31, 29-42. 
Moore, Gary C. and Izak Benbasat (1991), "Development of an Instrument to Measure the Perceptions of Adopting an Information Technology Innovation," Information Systems Research, 2 (3), 192-222.

Morris, Michael G. and Viswanath Venkatesh (2000), "Age Differences in Technology Adoption Decisions: Implications for a Changing Work Force," Personnel Psychology, 53 (2), 375-403.

Pagani, Margherita (2000), "Interactive Television: A Model of Analysis of Business Economic Dynamics," International Journal on Media Management, 2 (1), 25-36.

Papastathopoulou, Paulina, George J. Avlonitis, and Nikolas G. Panagopoulos (2007), "Intraorganizational Information and Communication Technology Diffusion: Implications for Industrial Sellers and Buyers,"Industrial Marketing Science, 36 (3), 322-36.

Pavlou, Paul A. and Mendel Fygenson (2006), "Understanding and Predicting Electronic Commerce Adoption: An Extension of the Theory of Planned Behavior," MIS Quarterly, 30 (1), 115-43.

Pelham, A.M. and D.T. Wilson (1996), "A Longitudinal Study of the Impact of Market Structure, Firm Structure, Strategy, and Market Orientation Culture on Dimensions of SmallFirm Performance," Journal of the Academy of Marketing Science, 24 (1), 27-43.

Plouffe, Christopher R., John S. Hulland, and Mark Vandenbosch (2001), "Research Report: Richness versus Parsimony in Modeling Technology Adoption Decisions-Understanding Merchant Adoption of a Smart Card-Based Payment System," Information Systems Research, 12 (2), 208-22.

Riemenschneider, Cindy K., David A. Harrison, and Peter P. Mykytyn (2002), "Understanding IT Adoption Decisions in Small Business: Integrating Current Theories," Information and Management, 40 (4), 269-85.

Rogers, Everett M. (1995). Diffusion of Innovation. New York: The Free Press.

Sahberwal, Rajiv, Anand Jeyaraj, and Charles Chowa (2006), "Information System Success: Individual and Organizational Determinants," Management Science, 52 (12), 1849-64.

Schepers, Jan and Martin Wetzels (2007), "A Meta-Analysis of the Technology Acceptance Model: Investigating Subjective Norms and Moderation Effects," Information and Management, 44 (1), 90-103.

Sheppard, Blair H., Jon Hartwick, and Paul R. Warshaw (1988), "The Theory of Reasoned Action: A Meta-Analysis of Past Research with Recommendations for Modifications and Future Research," Journal of Consumer Research, 15 (3), 325-43.

Singh, Jagdip and Gary Rhoads (1991), "Boundary Role Ambiguity in Marketing-Oriented Positions: A Multidimensional, Multifaceted Operationalization," Journal of Marketing Research, 28 (3), 328-38. 
Steenkamp, Jan-Benedict E.M. and Hans Van Trijp (1991), "The Use of LISREL in Validating Marketing Constructs," International Journal of Research in Marketing, 8 (4), 283-99.

Sun, Heshan and Ping Zhang (2006), "The Role of Moderating Factors in User Technology Acceptance," International Journal of Human-Computer Studies, 64 (2), 53-78.

Szajna, Bernadette (1996), "Empirical Evaluation of the Revised Technology Acceptance Model," Management Science, 42 (1), 85-92.

Tarafdar, Monideepa and Sanjiv D. Vaidya (2006), "Challenges in the Adoption of ECommerce Technologies in India: The Role of Organizational Factors," International Journal of Information Management, 26 (6), 428-41.

Tauder, Arthur R. (2005), "Getting Ready for the Next Generation of Marketing Communications," Journal of Advertising Research, 45 (1), 5-8.

Taylor, Shirley and Peter Todd (1995), "Understanding Information Technology Usage: A Test of Competing Models," Information Systems Research, 6 (2), 144-76.

Thompson, Ronald L., Christopher Higgins, and Jane M. Howell (1994), "Influence of Experience on Personal Computer Utilization: Testing a Conceptual Model," Journal of Management Information Systems, 11 (1), 167-87.

Tornatzky, Louis G. and Mitchell Fleischer (1990), The Process of Technological Innovation. Lexington, MA: Lexington Books.

Triandis, Harry C. (1977), Interpersonal Behavior. Monterey, CA: Brook/Cole.

Venkatesh, Viswanath, and Fred D. Davis (2000), "A Theoretical Extension of the Technology Acceptance Model: Four Longitudinal Studies," Management Science, 46 (2), 186-204.

---, Michael G. Morris, Gordon B. Davis, and Fred D. Davis (2003), "User Acceptance of Information Technology: Toward a Unified View," MIS Quarterly, 27 (3), 425-78.

Wirtz, W. and J. Schwartz (2001), "Strategic Implications of the Segment of One TV," International Journal on Media Management, 3, 15-25.

Workman, Michael (2005), "Expert Decision Support System Use, Disuse, and Misuse: A Study Using the Theory of Planned Behavior," Computers in Human Behavior, 21, 211-31.

Woszczynski, Amy B. and M.E. Whitman (2003), "The Problem of Common Method Variance in IS Research," in The Handbook of Information Systems Research, M.E. Whitman and Amy B. Woszczynski, eds. Hershey, PA: Idea Group Publishing, 66-77.

Wu, Ing-Long and Jian-Liang Chen (2005), "An Extension of Trust and TAM Model with TPB in the Initial Adoption of Online Tax: An Empirical Study," International Journal of Human-Computer Studies, 62 (2), 784-808. 
Yi, Mun Y., Joyce D. Jackson, Jae S. Park, and Janice C. Probst (2005), "Understanding Information Technology Acceptance by Individual Professionals: Toward an Integrative View," Information and Management, 43 (3), 350-63.

\author{
About the Authors \\ Verolien Cauberghe (Ph.D., University of Antwerp) is Assistant Professor of Marketing \\ Communication in the faculty of Political and Social Science, Ghent University. Her research \\ interests include advertising effectiveness, effectiveness of new ad formats, health \\ communication, crisis communication, and risk communication. E- \\ mail: verolien.cauberghe@ugent.be
}

Patrick De Pelsmacker (Ph.D., Ghent University, Belgium) is Professor of Marketing in the faculty of Applied Economics, University of Antwerp. His research interests include advertising effectiveness, branding, marketing communication via new media formats, and social marketing. E-mail: patrick.depelsmacker@ua.ac.be

\title{
Acknowledgments
}

The authors thank InSites, VMMa, and Pub Magazine for their operational support. Many thanks also go to the two scholars who carefully read and provided comments regarding revisions to this article. 